\title{
Upper limits on the gravitational mass loss of the Galaxy and the LIGO burst searches
}

\author{
Florian Dubath and Michele Maggiore \\ Département de Physique Théorique, Université de Genève, 24 quai Ansermet, CH-1211 Genève 4
}

(Dated: October 30, 2018)

\begin{abstract}
We discuss the relevance, for the search of gravitational-wave bursts, of upper limits on the total mass loss of the Galaxy which come from various astronomical observations. For sub-millisecond bursts we obtain limits on the event rate, as a function of the GW amplitude, which are stronger than the corresponding upper limits set by LIGO in the S2 run. A detection of a burst rate saturating these limits, with the sensitivities of present and near-future runs, would imply that, with some improvement on the accuracy of astronomical observations of the Galaxy, as foreseen with the GAIA mission, it might be possible to detect gravitational waves indirectly from their effect on galactic dynamics.
\end{abstract}

\section{INTRODUCTION}

One of the main target of the existing gravitationalwave $(\mathrm{GW})$ detectors are short bursts, with a duration between fractions of a millisecond and a few seconds, which could originate from astrophysical events. The LIGO collaboration has published the results of the S2 run [1], (see also [2] for the S3 run) which extended the search to lower values of the GW amplitude, compared to previous searches by resonant-mass detectors [3, 4, 5]. Presently, LIGO is performing a long data-taking run at its target sensitivity which, in case of no positive detection, should anyway produce a more significant bound on the event rate.

The result of these searches can be presented as an upper limit on the event rate of GW bursts, $\mathcal{R}$, as a function of the strength of the GW signal. The latter can be conveniently characterized in terms of the so-called root-sum-square amplitude $h_{\text {rss }}$, defined by

$$
h_{\mathrm{rss}}^{2} \equiv \int_{-\infty}^{\infty} d t h^{2}(t) .
$$

The purpose of this note is to point out that a bound on $\mathcal{R}$ as a function of $h_{\text {rss }}$ can also be obtained from considerations of galactic dynamics, and it is in fact quite significant compared to the bounds that can be obtained with existing and near-future sensitivities of GW detectors, especially for bursts of short duration, say $\tau \simeq 0.1 \mathrm{~ms}$.

The bound emerges from the fact that, if there is a steady rate of GW bursts, the Galaxy has a corresponding rate of mass loss into GWs. Since it is difficult to imagine that the burst rate today is significantly higher than in the past, one must consider the cumulative effect of this mass loss over a period comparable to the age of the Galaxy, and this can have significant consequences on various aspects of galactic dynamics. These issues were first addressed many years ago [6, 7, [8, 9], and in [10] we reconsidered them, using the present knowledge of galactic dynamics, and we showed that much more stringent bounds emerge nowadays. Let us summarize the results, referring the reader to Ref. [10] for details and more references. The main observations that allow us to put a bound are discussed below.

\section{UPPER LIMITS FROM GALACTIC DYNAMICS}

(i) Effect of the mass loss on the radial velocity of stars. If the Galaxy is loosing mass, stars become less and less bound and acquire radial velocities with respect to the Galaxy rest frame. This modifies the radial velocity of stars, $v_{r}$, inducing a so-called $K$-term,

$$
v_{r}=A R \sin 2 l+K R,
$$

where the term $A R \sin 2 l$ is the standard effect due to the differential rotation of the Galaxy ( $A$ is Oort's constant, $R$ is the distance of the star from the sun, and $l$ the galactic longitude), and the effect of mass loss is in the second term, where $K=-\dot{M} / M$, and $M$ is the mass of the Galaxy. From the experimental bound on $K$, we deduced in [10] a bound

$$
-\dot{M}<O(30) M_{\odot} / \text { yr . }
$$

(ii) Mass loss and outward motion of the LSR. Rather than looking at the $K$ term, i.e. at the expansion/contraction of the stars within a few kpc from the sun, one can investigate whether the local standard of rest (LSR) has an overall outward radial velocity, as suggested by eq. (2). Here, the most interesting information comes from the observation of the $21 \mathrm{~cm}$ absorption line toward the galactic center [11], which shows that the gas along the line-of-sight has a mean radial velocity with respect to the LSR of $-0.23 \pm 0.06 \mathrm{~km} / \mathrm{s}$. The absorbing material is probably at $1-2 \mathrm{kpc}$ from the galactic center. A radial expansion due to mass loss predicts a radial velocity $v_{r} \sim r$, see eq. (2), where $r$ is the radial distance from the galactic center, and therefore we should expect a difference in velocity between us and this gas, $\Delta v_{r}=(-\dot{M} / M) \Delta r$, where $\Delta r \simeq 6 \mathrm{kpc}$ is the distance of the Sun from the absorbing gas.

In general, there can be both positive and negative contributions from different physical mechanisms to the 
value $\Delta v_{r}=-0.23 \pm 0.06 \mathrm{~km} / \mathrm{s}$, and to extract a bound on mass loss to GW we require that no fine tuning between different contributions takes place. We set conventionally at $20 \%$ the maximum fine tuning that we allow, which means that we say that a positive contribution from GWs to $\Delta v_{r}$, if it exists at all, must be smaller than $O(0.04) \mathrm{km} / \mathrm{s}$. Setting the distance between us and the gas to $\Delta r=6 \mathrm{kpc}$, this gives a bound

$$
-\dot{M}<O(0.5) M_{\odot} / \mathrm{yr}
$$

Of course, precise numbers depend on the level of fine tuning that we can tolerate, but it is clear that we cannot raise this bound by, say, one order of magnitude, without invoking very unnatural cancellations between completely unrelated phenomena.

(iii) Upper limits from globular clusters. Similar bounds have been found using globular clusters as probes [8, 12]. The idea is that, if the mass of the Galaxy was much bigger in the past, the orbits of globular clusters would have been much closer to the galactic nucleus, and this close interaction with a very massive central nucleus would have produced the tidal disruption of the cluster. The analysis of five different globular clusters gives the result 12 ]

$$
-\dot{M}<O(10) M_{\odot} / \text { yr } .
$$

(iv) Old wide binaries. Another limit comes from the existence of old wide binaries, since for a very massive galactic nucleus the galactic orbits would have been much smaller than at present. Therefore the density of stars would have been much larger and the dissolution time of binaries due to stellar encounters correspondingly shorter. From a list of 11 well observed old wide binaries one finds a limit on steady mass loss [8]

$$
-\dot{M}<O(10) M_{\odot} / \mathrm{yr} .
$$

In conclusion, we have four different methods which all give a bound on $-\dot{M}$ between $O(1)$ and $O(10) M_{\odot}$, so we write

$$
-\dot{M}<\epsilon M_{\odot} / \mathrm{yr}
$$

and we expect $\epsilon \sim 1$, unless one cannot find a way, perhaps with some fine tuning, to relax the most stringent bound (4). Anyway, given that we have three more independent limits on $\epsilon$, we see that we cannot stretch the value of $\epsilon$ beyond, say, $\epsilon \simeq 10$. Furthermore, independently of the technical details leading to the above bounds, it is easy to understand qualitatively why a bound on $-\dot{M}$ of order $1 M_{\odot} /$ yr emerges. The total mass of the galactic disk plus bulge and spheroid is estimated to be $9 \times 10^{10} M_{\odot}$, while a lower bound on the age of the Galaxy is provided by the age of its oldest globular clusters, which is $1.2 \times 10^{10} \mathrm{yr}$. Therefore a mass loss rate due to GWs of the order of a few solar masses per year implies a total mass loss, over the age of the Galaxy, comparable to its present mass. It is not surprising that, at this level, one finds important dynamical effects related mass loss. If one streched $\epsilon$ up to values of order 10, one should admit that the mass that the galactic disk has lost to GWs during its history is larger than its present mass, so over $50 \%$ of the original mass would have been lost to GWs.

We also remark that these are just upper bounds and, as far as the above arguments are concerned, there is no physical reason that suggests that they might be almost saturated, so they should not be taken as an indication of a plausible value of $\dot{M}$.

\section{IMPLICATIONS FOR GALACTIC BURSTS OF GWS}

We now compare this bound, which is generic and holds whatever is the physical origin of the mass loss of the Galaxy, with the energy carried away by GWs, if there are GW bursts with a typical rss amplitude $h_{\text {rss }}$ and a rate $\mathcal{R}$. Following Ref. [1], we consider a Gaussian waveform of duration $\tau$, given by

$$
h(t)=h_{\mathrm{rss}}\left(\frac{2}{\pi \tau^{2}}\right)^{1 / 4} e^{-t^{2} / \tau^{2}}
$$

whose Fourier transform is

$$
\tilde{h}(f)=h_{\mathrm{rss}}\left(2 \pi \tau^{2}\right)^{1 / 4} \exp \left(-\pi^{2} \tau^{2} f^{2}\right) .
$$

(The same analysis can be repeated with sine-gaussian waveforms, with similar results). We first consider a wave coming from optimal direction with $h_{+}(t)=h(t)$ and $h_{\times}(t)=0$. From the standard expression of the energy flux,

$$
\frac{d E}{d A d f}=\frac{\pi c^{3}}{2 G} f^{2}\left(\left|\tilde{h}_{+}(f)\right|^{2}+\left|\tilde{h}_{\times}(f)\right|^{2}\right),
$$

where $d A=r^{2} d \Omega$ and $r$ is the distance to the source, we get that the total energy radiated by such a burst,

$$
E=4 \pi r^{2} \int_{0}^{\infty} \frac{d E}{d f}=\frac{r^{2} c^{3} h_{\mathrm{rSS}}^{2}}{4 G \tau^{2}}
$$

The average over the square of the pattern function of the interferometer gives the usual factor $2 / 5$ which, following the conventions of the LIGO collaboration, we include in the definition of $h_{\mathrm{rss}}$. Then, the energy carried by a burst, averaged over the arrival direction and the polarization, is

$$
\begin{aligned}
\langle E\rangle= & \frac{r^{2} c^{3} h_{\mathrm{rss}}^{2}}{4 G \tau^{2}} \simeq 3.4 \times 10^{-4} M_{\odot} c^{2}\left(\frac{r}{8 \mathrm{kpc}}\right)^{2} \\
& \times\left(\frac{h_{\mathrm{rss}}}{10^{-19} \mathrm{~Hz}^{-1 / 2}}\right)^{2}\left(\frac{1 \mathrm{~ms}}{\tau}\right)^{2}
\end{aligned}
$$

where in the second line we normalized $r$ to the distance to the galactic center. We see from the above that a burst 


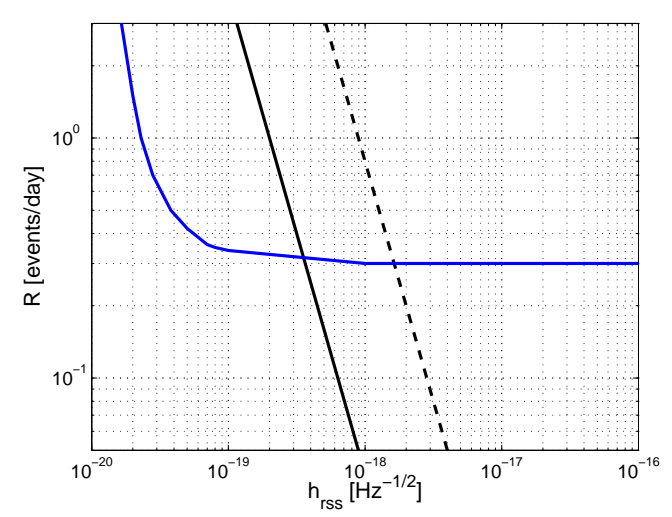

FIG. 1: The limit from galactic dinamics with $\epsilon=0.5$ (solid line) and $\epsilon=10$ (dashed), compared to the bound from the LIGO S2 run, when $\tau=1$ ms. LIGO data taken from Fig. 12 of Ref. [1].

of duration $1 \mathrm{~ms}$, with $h_{\mathrm{rss}}=10^{-19} \mathrm{~Hz}^{-1 / 2}$, carries away about $3 \times 10^{-4}$ solar masses in GWs, if it comes from a source located at typical galactic distances. Of course shorter bursts are more energetic, since they extend in frequency space up to $f_{\max } \sim 1 / \tau$. In an astrophysical context it makes sense to consider bursts as short as $\tau \simeq 0.1 \mathrm{~ms}$, corresponding, for the gaussian wavepacket (9), to $f_{\max }$ of order of a few $\mathrm{kHz}$, which indeed are the shortest burst searched for in Refs. 1, 2]. If $\mathcal{R}$ is the rate of these bursts, the associated rate of mass loss of the Galaxy is $\dot{M}=-\mathcal{R} \times\langle E\rangle / c^{2}$, with $\langle E\rangle$ given by eq. (12). Combining this with eq. (17) we get

$\mathcal{R}<8.0 \epsilon \frac{\text { events }}{\text { day }}\left(\frac{8 \mathrm{kpc}}{r}\right)^{2}\left(\frac{10^{-19} \mathrm{~Hz}^{-1 / 2}}{h_{\mathrm{rss}}}\right)^{2}\left(\frac{\tau}{1 \mathrm{~ms}}\right)^{2}$,

where now $r$ is an average distance scale characterizing the population of sources [15]. In Fig. 11 we show this bound, in the $\left(\mathcal{R}, h_{\mathrm{rss}}\right)$ plane, for $\tau=1 \mathrm{~ms}, r=8 \mathrm{kpc}$, and we compare it to the experimental bounds obtained by the S2 LIGO run, for the same value of $\tau$. The solid line corresponds to $\epsilon=0.5$, and the dashed line to $\epsilon=10$. In Fig. 2]we perform the same comparison for $\tau=0.1 \mathrm{~ms}$.

We see that the bound from galactic dynamics is a significant one, especially for small $\tau$, and indeed for $\tau=0.1 \mathrm{~ms}$ is quite stronger than the LIGO S2 result. With the on-going and future high-sensitivity runs by LIGO and VIRGO one can expect that, even for $\tau=0.1 \mathrm{~ms}$, one will go beyond the small- $h_{\mathrm{rss}}$ portion of the limiting curve determined from galactic dynamics, while at larger $h_{\text {rss }}$ the limit from galactic dynamics will remain the dominant one. If a statistically significant rate in excess of the value obtained from eq. (13) with $\epsilon \simeq 1$ and $r \simeq 8 \mathrm{kpc}$ were found and if, from the energetic of the events, one concluded that they originated in our Galaxy, then one should study the possibility of relaxing somehow the bound on $\epsilon$ (which anyway should be possible at most by one order of magnitude). Otherwise,

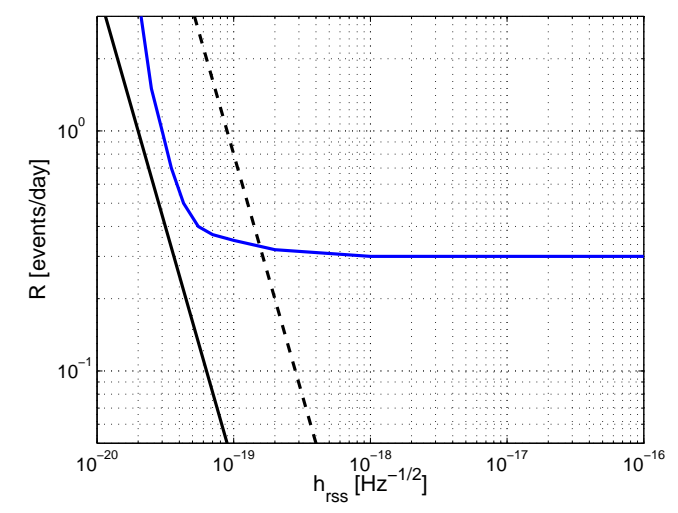

FIG. 2: The same as Fig. 1, with $\tau=0.1 \mathrm{~ms}$.

one could consider the possibility that, rather than having a homogeneous distribution of sources in the galactic disk, the signal could be due to a single (or a few) source at a close distance from us, which emits repeatedly GW bursts [10, 13]. Therefore, eq. (13) can give useful clues as to the spatial distribution and possibly the origin of the sources.

Conversely, if GW detectors should find that the bound that we have discussed is saturated, this would mean that the effect of GW emission on some astronomical observables, such as the radial motion of the LSR, is just of order $10-20 \%$ of the present observational uncertainties. This would imply that, with an increase in the accuracy of astronomical observations, as is expected with the GAIA mission (see e.g. Ref. 14]), one could be able to single out the effect of GW emission on the dynamics of the Galaxy, providing a form of indirect detection of GWs.

Our work is partially supported by the Fond National Suisse. We thank Erik Katsavounidis for useful comments.
[1] B. Abbott et al. (LSC), Phys. Rev. D 72, 062001 (2005).

[2] B. Abbott et al. (LSC), arXiv:gr-qc/0511146

[3] P. Astone et al., (ROG) Class. Quant. Grav. 19 (2002) 5449.

[4] P. Astone et al. (IGEC), Phys. Rev. D 68, 022001 (2003)
[5] P. Astone et al., (ROG) in Proceeding of the 6th Amaldi Conference, Okinawa (Japan) 2005, to appear in Class. Quant. Grav.

[6] F.J. Kerr, MNRAS 123 (1962) 327.

[7] D. Sciama, G. Field and M. Rees, Phys. Rev. Lett. 23 
(1969) 1514.

[8] A. Poveda and C. Allen, ApJ 200 (1975) 42.

[9] M.W. Ovenden and J. Byl, ApJ 206 (1976) 57.

[10] E. Coccia, F. Dubath, and M. Maggiore Phys. Rev. D 70 (2004) 084010.

[11] V. Radhakrishnan and V.N.G. Sarma, MNRAS 85 (1980) 249.

[12] F. Dubath and A. Rissone, Class. Quant. Grav. 21 (2004) 5075 .

[13] F. Dubath, S. Foffa, M. A. Gasparini, M. Maggiore and R. Sturani, Phys. Rev. D 71 (2005) 124003.
[14] M. A. C. Perryman et al., Astron. Astrophys. 369 (2001) 339.

[15] More precisely, we are considering an ensemble of bursts such that the average of the square of their rss amplitude is $h_{\mathrm{rss}}^{2}$, and $r^{2}$ is the average of their squared distance. We are also assuming an isotropic distribution of sources, since $\langle E\rangle$ is averaged over all directions in the sky. This assumption should be correct to within a factor of order unity, which is not a significant error for the order-ormagnitude estimates in this paper. 